\title{
The State of the Art in Microseismic Monitoring of Tailings Dams: Reduced and Field- scale Experiments
}

Igor Lopes Santana Braga*1, Fernando Sergio Moraes ${ }^{3,1}$, Igor Barbosa de Oliveira ${ }^{1}$, Alan Cunha², Marco Antonio Braga ${ }^{2}$

${ }^{1}$ Invision Geophysics, ${ }^{2}$ CPGA/UFRJ, ${ }^{3}$ LENEP/UENF

Copyright 2021, SBGf - Sociedade Brasileira de Geofísica.

This paper was prepared for presentation during the $17^{\text {th }}$ International Congress of the Brazilian Geophysical Society held in Rio de Janeiro, Brazil, $16-19$ August 2021.

Contents of this paper were reviewed by the Technical Committee of the $17^{\text {th }}$ International Congress of the Brazilian Geophysical Society and do not necessarily represent any position of

the SBGf, its officers or members. Electronic reproduction or storage of any part of this paper for commercial purposes without the written consent of the Brazilian Geophysical Society is prohibited.

\begin{abstract}
The social and environmental impacts of the recent accidents involving tailings dams rupture in Brazil motivated a search for new technologies that can improve the risk management of these structures. Geophysical methods are suitable as non-invasive and cost-effective tools. A recent effort held by the industry and academy to expand geophysical technologies resulted in a complete microseismic solution for characterizing and monitoring dams internal structure.
\end{abstract}

The microseismic monitoring system consists of a set of innovative methodologies, hardware and software. Different types of microseismic data and processing workflows can assist in the safety and stability of dams and mining pits. The current system comprises sensor node units, front and backend applications. Two microseismic monitoring strategies, conventional and passive, are supported. Conventional microseismic represents the analysis of triggered events to determine their source location and attributes used as stability indicators for the monitored structure. The events need to be identified and isolated, which requires continuous data analysis and waveform registration. The passive microseismic monitoring is based on ambient noise recording to assess the change in the velocity of propagation of seismic waves, which may indicate zones of instability in the monitored location. The interferometry technique is applied to estimate the variation in the medium's velocity from the periodic recording of the microseismic noise.

The presentation describes reduced and field-scale experiments used to test the system. The reduced-scale experiment utilizes a physical model of a tailings dam constructed over an unconsolidated quaternary fine gray sandstone. A homogeneous shaly material is used to build a square foundation of a side measuring $810 \mathrm{~cm}$ and the walls. The dam walls reach a crest $50 \mathrm{~cm}$ height and $30 \mathrm{~cm}$ width, with a slope of $2: 1(\mathrm{H}: \mathrm{V})$. In addition to the two microseismic monitoring techniques described above, a third method using active source MASW (Multichannel Analysis of Surface Wave) is used to characterize the dam site. Two drill holes provide the ground truth, given by the same fine sandstone with different compaction levels as verified by the SPT (Standard Penetration Test) data.

Following the reduced-scale experiment, a real dam monitoring experiment has been arranged to start in the second semester of 2021. Inspection and monitoring of tailings dams traditionally include visual inspection and direct measurements using conventional equipment: displacement meter, water level meter, flow meter, piezometers. The located nature of these measurements brings some limitations for volume analysis, and this dataset cannot map weak areas and unexpected saturated spots existing out the investigation points. By jointly interpreting the microseismic and geotechnical data, such limitations can be eliminated and more meaning can be attached to the microseismic data interpretation.

The development of a national microseismic system represents a significant advance for the Brazilian industry, aiming to provide safety to national mining operations while minimizing the external dependence of this technology. 